\title{
Spectral characteristics of waves and particles in the model of cyclotron wave-particle interactions near plasmapause
}

\author{
D. L. Pasmanik and V. Y. Trakhtengerts \\ Institute of Applied Physics, Ulyanov Street 46, Nizhny Novgorod 603600, Russia
}

Received: 30 April 1998 / Accepted: 17 July 1998

\begin{abstract}
Further analysis of energetic electron precipitation at the evening sector of magnetosphere is performed. In the framework of the quantitative model of cyclotron wave-particle interactions developed in the previous Pasmanik et al. paper, the case of finite spread over energies of initial energetic electron distribution is studied. The solution for distribution function of energetic electron is found. The energetic spectrum of trapped and precipitating electrons and whistler wave spectrum are analysed.
\end{abstract}

Key words. Magnetospheric physics (energetic particles, precipitating; energetic particles, trapped); S Space plasma physics (wave-particle interactions).

\section{Introduction}

This work is devoted to the problem of the cyclotron wave-particle interactions in the magnetosphere. In particular, the quantitative model for whistler mode wave and energetic particle interactions in the Earth's radiation belts with real particle and wave sources taken into account is studied. The development of such a model is really important, because many experimental data on energetic particles and waves in the magnetosphere have now been accumulated.

In a previous paper (Pasmanik et al., 1998) the formation of a zone of energetic electron precipitation during magnetic storm was analysed in the framework of the quasi-linear theory of cyclotron instability. As a source of energetic particles Pasmanik et al. (1998) considered the magnetic drift, following injection at the nightside of the magnetosphere. However, it was

Correspondence to: D. L. Pasmanik e-mail: pdl@aurora.appl.ssi-nnov.ru thought that the injected electrons had a small spread over energies and the wave spectrum was not analysed.

In this work we have performed further development of that model. In particular, we have generalised previously obtained results for the case of arbitrary initial distribution of energetic particles over energy and found the spectrum of generated waves.

The work is organised as follows: the basic equations of the developed model are presented in Sect. 2, their analytical solution is given in Sect. 3 and results of numerical analysis of this solution are discussed in Sect. 4 . A conclusion is formulated in Sect. 5.

\section{Basic equations}

Let us consider the development of cyclotron instability due to the interactions between whistler mode waves and energetic electrons, which enter the region with enhanced background plasma density by their longitudinal drift. Such a region may be found at the plasmapause or when detached cold plasma clouds formed on the recovery phase of magnetic storm.

As basic equations we use the set of self-consistent equations of the quasi-linear theory of cyclotron instability, including the equation for distribution function of energetic electrons $F$ and spectral density $\varepsilon_{\omega}$ of whistler mode waves. In the case of dense background plasma when the parameter $\beta_{\star}=\omega_{p L}^{2} v_{0}^{2} / \omega_{B L}^{2} c^{2} \gg 1$, most wave energy occurs at low frequencies $\omega \sim \omega_{B L} / \beta_{\star} \ll \omega_{B L}$ and pitch angle diffusion prevails. Here $\omega_{B L}$ and $\omega_{p L}$ are the electron gyro- and plasma frequency at the equatorial plane, $v_{0}$ is characteristic velocity of energetic electrons, $c$ is the velocity of light. In this case the basic equations can be written in the following form (Pasmanik et al., 1998):

$\Omega_{D} \frac{\partial F}{\partial \varphi}=\frac{1}{T_{b}} \frac{\partial}{\partial \mu} \mu D \frac{\partial F}{\partial \mu}-\delta \cdot F$

$v_{g \perp} \frac{\partial \varepsilon_{\omega}}{\partial r_{\perp}}=(\gamma-v) \varepsilon_{\omega}$, 
The first equation is averaged over electron bounce oscillations between magnetic mirror points. The term on the left side in Eq. (1) describes the longitudinal magnetic drift of energetic electrons, where $\varphi$ is the azimuthal angle, $\Omega_{D}$ is angular magnetic drift velocity, $T_{b}=\oint \mathrm{d} z / v_{\|}$is electron bounce period, $v_{\|}$and $z$ are velocity components and coordinates along magnetic field line. Magnetic moment $\mu=\sin ^{2} \Theta_{L}$, where $\Theta_{L}$ is pitch angle at the equatorial plane. The last term in Eq. (1) corresponds to the particle losses through the loss cone, the coefficient $\delta$ is defined as

$\delta=\left\{\begin{array}{ll}0 & \mu \geq \mu_{c} \\ \delta_{0}=v / l & 0 \leq \mu \leq \mu_{c}\end{array}\right.$,

where $\mu_{c}=L^{-3}\left(4-3 L^{-1}\right)^{-1 / 2}$ is the loss cone boundary for the dipolar magnetic field, $v$ is the electron velocity, and $l$ is the length of the magnetic flux tube. The diffusion coefficient $D$ is determined by the wave intensity $\varepsilon_{\omega}$ and can be written as (Pasmanik et al., 1998):

$D=V\left(v^{2}\right) \tilde{D}=V \cdot \int_{\omega_{m 0}}^{\omega_{B L}} G_{1} \varepsilon_{\omega} \mathrm{d} \omega$,

where,

$V=\left(\frac{4 \pi e}{m c v}\right)^{2}, G_{1}=\frac{l_{\text {eff }}}{k}$.

Here $k$ is the wave vector of whistler wave and $l_{\text {eff }}$ is the effective length of the whistler-electron interaction region near the equator; generally it depends on wave frequency and electron velocity (see Bespalov and Trakhtengerts, 1986), but under the conditions mentioned here we may consider $l_{\text {eff }} \approx$ const.

In the energy transfer Eq. (2) the term $\gamma_{\varepsilon_{\omega}}$ describes the energy source for whistler waves, and $\gamma$ is the growth rate of the cyclotron instability. Two other terms corresponds to losses due to the imperfect reflection from the ionosphere (the term $v \varepsilon_{\omega}, v$ is the effective damping rate) and wave propagation away from the generation region across the magnetic field due to refraction (the term $v_{g \perp} \frac{\partial \varepsilon_{\omega}}{\partial r_{\perp}}$ ). In Eq. (2) $v_{g \perp}$ and $r_{\perp}$ are the components of whistler mode group velocity and the spatial coordinate perpendicular to the magnetic field respectively. It should be remembered that the main propagation term along the magnetic field $v_{g \|} \frac{\partial \varepsilon_{\omega}}{\partial z}$ has been removed by averaging over the oscillations of wave-packets between conjugate ionospheres.

Further, we consider a one-dimensional problem and make $\mathrm{d} r_{\perp}=R_{0} L \mathrm{~d} \varphi$, where $R_{0}$ is the Earth's radius.

The growth rate $\gamma$ is found from the linear theory of cyclotron instability and can be written as (Bespalov and Trakhtengerts, 1986),

$\gamma \approx \frac{1}{T_{g}} \frac{(2 \pi)^{3} e}{c B_{L}} \int_{\omega_{B L} / k}^{\infty} \int_{0}^{\mu_{m}} l_{\text {eff }} \mu v^{3}\left(\frac{\partial F}{\partial \mu}-\frac{\omega}{\omega_{B L}} F\right) \mathrm{d} \mu \mathrm{d} v$,

where $\mu_{m}=1-\left(\omega_{B L} / k v\right)^{2}, T_{g}=\oint \mathrm{d} z / v_{g \|}$ is period of wave-packet oscillations between conjugate ionospheres, and the electron distribution function is normalised by the relation:

$N=\pi \mu_{c}^{-1} \int_{0}^{\infty} \int_{0}^{1} T_{b} F v^{3} \mathrm{~d} \mu \mathrm{d} v$.

Here $N$ is the number of electrons in the magnetic flux tube with unit cross-section in the ionosphere.

The self-consistent system of Eq. (1) and (2) must be completed by the boundary conditions, which take the form:

$$
\begin{aligned}
& \varphi=0: \varepsilon_{\omega}=\varepsilon_{\omega 0}, F=F_{0}(\mu) \\
& \mu=0 ; 1: \mu \frac{\partial F}{\partial \mu}=0 .
\end{aligned}
$$

\section{Solution in the cases of strong and weak diffusion}

Following Pasmanik et al. (1998) we shall consider two cases corresponding to strong and weak pitch angle diffusion. The criterion of diffusion regime is ratio of the loss cone filling time in the process of pitch angle diffusion to the time required to empty the loss cone. This ratio is equal to

$K \equiv \frac{D}{\mu_{c} T_{b} \delta_{0}} \approx \frac{D}{1.5 \mu_{c}}$

$K \gg 1$ for strong diffusion and $K \ll 1$ in the weak diffusion case.

According to Pasmanik et al. (1998) in both cases solution of Eq. (1) is found from the equation without loss term $\delta F$

$\frac{\partial \tilde{F}}{\partial \varphi}=\frac{V \tilde{D}}{\Omega_{D} T_{b}} \frac{\partial}{\partial \mu} \mu \frac{\partial \tilde{F}}{\partial \mu}$.

where $V$ and $\tilde{D}$ are determined by Eq. (5). The losses are taken into account by the multiplier $\exp (-\Delta \cdot \varphi)$ in the storng diffusion case, where $\Delta=\delta_{0} \mu_{c} / \Omega_{D}$, and by changing boundary condition for Eq. (10) at the edge of loss cone $\mu=\mu_{c}$, which is written as $F\left(\mu=\mu_{c}, \varphi\right)=0$ in the case of weak diffusion.

In contrast to Pasmanik et al. (1998), we suggest a finite spread of initial distribution function over velocity. Then we have to consider the dependencies of parameters in Eq. (10) on velocity. Taking into account that $\Omega_{D} \propto v^{2}$, and $T_{b} \propto v^{-1}$ (see Lyons and Williams, 1984) we obtain:

$\frac{\partial F}{\partial \xi}=W(v) \frac{\partial}{\partial \mu} \mu \frac{\partial F}{\partial \mu}$.

where $W(v)=v_{0}^{3} / v^{3}$; the new variable $\xi$ is defined by the relation

$d \xi=\Theta(\varphi) d \varphi$

the coefficient $\Theta(\varphi)=\left(D / \Omega_{D} T_{b}\right)_{v=v_{0}}, v_{0}$ is the velocity characteristic value. 
The solution of Eq. (11) can be written as

$F=\sum_{k} A_{k}(v) Z_{k}(\mu) \exp \left(-\lambda_{k} W(v) \xi\right)$,

where $Z_{k}$ and $\lambda_{k}$ are eigenfunctions and eigenvalues of diffusion operator. The sets of $Z_{k}$ and $\lambda_{k}$ depend on the diffusion regime (Pasmanik et al., 1998). The coefficient $A_{k}$ is given by:

$A_{k}(v)=\left(\int_{0}^{1} F_{0}(\mu, v) Z_{k}(\mu) \mathrm{d} \mu\right)\left(\int_{0}^{1} Z_{k}^{2}(\mu) \mathrm{d} \mu\right)^{-1}$

and depends here on $v$.

Substituting the solution for distribution function (13) into Eq. (6) we obtain the following expression for the growth rate $\gamma(\xi, \omega)$ :

$\gamma=\gamma_{0} \sqrt{\frac{\omega}{\omega_{B L}}}\left(B_{1}(\xi)-\frac{\omega}{\omega_{B L}} B_{2}(\xi)\right)$,

where

$\gamma_{0}=\pi \frac{l_{\mathrm{eff}}}{n_{c L}} \frac{\omega_{p}}{c}$

$B_{1}(\xi)=\sum_{k} \int_{0}^{1} \mu \frac{\partial Z_{k}(\mu)}{\partial \mu} \mathrm{d} \mu \int_{0}^{\infty} A_{k}(v) v^{3} \mathrm{e}^{-\lambda_{k} W(v) \xi} \mathrm{d} v$,

$B_{2}(\xi)=\sum_{k} \int_{0}^{1} \mu Z_{k}(\mu) \mu d \mu \int^{\infty} A_{k}(v) v^{3} \mathrm{e}^{-\lambda_{k} W(v) \xi} d v$.

Here we took into account that for $\omega \ll \omega_{B L}$ the wave vector for whistler waves is written as

$k^{2} \approx \frac{\omega \omega_{p}^{2}}{\omega_{B} c^{2}}$,

and $n_{c L}$ is the plasma density at the equatorial plane.

The expression for spectral wave density $\varepsilon_{\omega}$ can be found by integrating Eq. (2) and is written in the form

$\ln \frac{\varepsilon_{\omega}}{\varepsilon_{\omega 0}}=C_{1}(\xi)\left(\frac{\omega}{\omega_{B L}}\right)^{\frac{1}{2}}-C_{2}(\xi)\left(\frac{\omega}{\omega_{B L}}\right)^{\frac{3}{2}}-v \varphi(\xi)$,

where

$C_{1,2}(\xi)=\gamma_{0} \Omega_{D} T_{b} \int_{0}^{\xi} \frac{B_{1,2}(\xi)}{D} d \xi$.

This solution can be used to find the diffusion coefficient $D(\varphi)$. But the equation for $D(\varphi)$ which appears after substitution of Eqs. (20)-(21) into Eq. (4) is too complicated. To simplify this equation we use some properties of the spectral density $\varepsilon_{\omega}$ and growth rate $\gamma$, which follow from their dependencies on frequency.

According to Eqs. (15) and (20) the functions $\varepsilon_{\omega}(\omega)$ and growth rate $\gamma(\omega)$ have one maximum. From numerical analysis we found that the growth rate depends on the frequency more smoothly then the wave spectral density while the locations of maxima are close to each other. Hence, integrating both parts of Eq. (2) over $\omega$ with the weighting function $G_{1}$ and using Eq. (4) it is possible to obtain a simplified equation for the diffusion coefficient $D$. Taking into account the properties of $\varepsilon_{\omega}$ and $\gamma$ discussed we may carry $\gamma$ away from the integral, and consider its value at the maximum: $\omega=\omega_{m}(\xi)=\omega_{H L} B_{1} / 3 B_{2}$. The resulting equation has the form

$\frac{v_{g \perp}}{R_{0} L} \frac{\partial D}{\partial \varphi}=\left(\gamma_{m}-v_{0}\right) D$,

where $\gamma_{m}=\frac{2 \gamma_{0}}{3}\left(\frac{B_{1}^{3}}{3 B_{2}}\right)^{\frac{1}{2}}$ and $v_{0}$ are values under $\omega=\omega_{m}$. Using the expression (12) this equation is reduced to

$\frac{\partial \Theta}{\partial \xi}=\alpha\left(\gamma_{m}-v_{0}\right)$,

with $\alpha=R_{0} L / v_{g \perp}$, and its solution

$\Theta(\xi)=\Theta_{0}+\int_{0}^{\xi} \gamma_{m}(\xi) \mathrm{d} \xi-\alpha v_{0} \xi$,

where $\Theta_{0}=\left(D_{0} / \Omega_{D} T_{b}\right)_{v=v_{0}}$ defined from the initial conditions. This expression may be used to acquire dependence $\varphi(\xi)$.

\section{Numerical analysis}

This section devoted to the numerical analysis of solution of Eq. (13), (15), (20) and (24).

In the numerical simulations we examined evolution of the energetic electron distribution over velocities $\left(F_{v}\right)$, the wave spectral density $\left(\varepsilon_{\omega}\right)$ and fluxes of trapped $\left(S_{t r}\right)$ and precipitating $\left(S_{p r}\right)$ particles along the longitudinal coordinate $\varphi$. The particle fluxes are defined as following (Pasmanik et al. 1998): the flux density of precipitating particles is equal to

$S_{p r}^{S}=2 \pi \int_{0}^{\infty} F^{S}\left(\mu_{c}, v\right) v^{3} \mathrm{~d} v$

in the case of strong diffusion and

$S_{p r}^{W}=\pi \int_{0}^{\infty}\left(D \frac{\partial F^{W}}{\partial \mu}\right)_{\mu_{c}} v^{3} \mathrm{~d} v$

in the case of weak diffusion; the flux density of trapped particles is

$S_{t r}^{S, W}=2 \pi b \int_{0}^{\infty} v^{3} \mathrm{~d} v \int_{\mu_{c}}^{1 / b} F^{S, W}(\mu, v) \mathrm{d} \mu$

in both cases.

The initial distribution function of energetic electrons was chosen in the form

$F_{0}(\mu, v)=\beta\left(\mu-\mu_{c}\right) \cdot \exp \left(\frac{\left(v-v_{0}\right)}{\Delta v}\right)^{2}$ 
where $\beta \approx S_{0 L} \pi^{-\frac{1}{2}}\left(v_{0}^{3} \Delta v\right)^{-1}$ is the normalising constant, and $S_{0 L}$ is the initial flux of energetic electrons at the equatorial plane.

The evolution of the distribution function of trapped particles over velocity at the equatorial plane is shown in Figs. 1 and 2 for the cases of strong and weak diffusion respectively. It is seen, that in both cases of strong and weak diffusion the amplitude of the distribution function is decreasing, with some shift of maximum location. The location of maximum is shifting to bigger values of

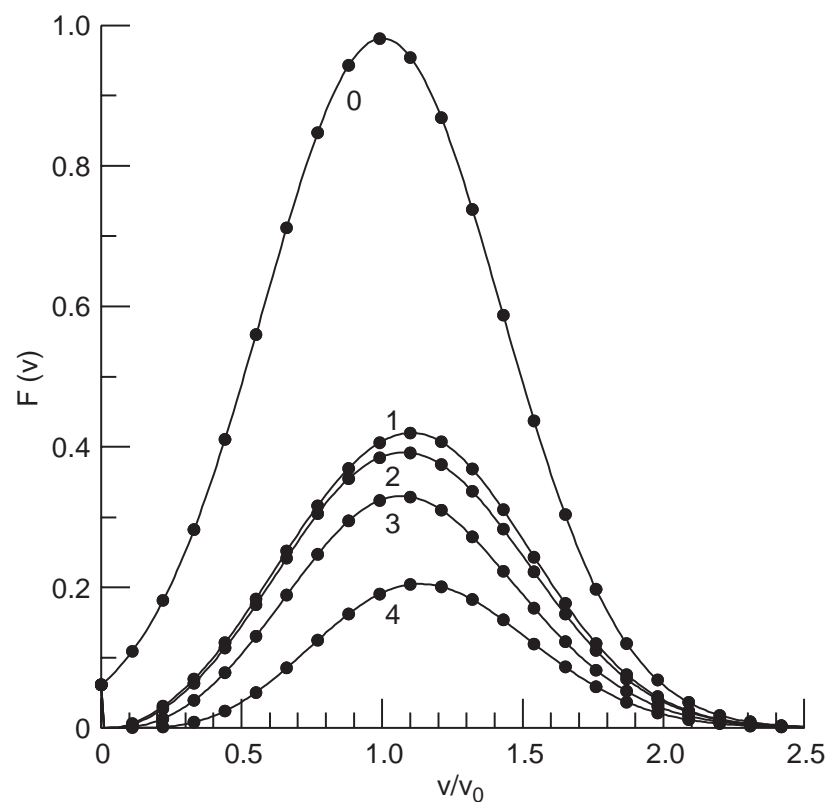

Fig. 1. The transformation of energetic electrons distribution over energy $F(v)$ with increase in $\varphi$ in the strong diffusion case at equatorial plane. $\Delta v / v_{0}=0.6, \quad m v_{0}^{2} / 2=50 \mathrm{keV}, \quad \mu_{c}^{-1}=160$, $D_{\max } \mu_{c}^{-1} \approx 8, \quad v_{g \perp}=10^{2} \mathrm{~km} / \mathrm{s}, \ln \left(D_{m} / D_{0}\right) \approx 10, v_{0} / \gamma_{m}(0)=0.01$, $\varphi_{0}=0, \varphi_{1} \approx 0.18^{\circ}, \varphi_{2} \approx 0.2^{\circ}, \varphi_{3} \approx 0.32^{\circ}, \varphi_{4} \approx 0.5^{\circ}$

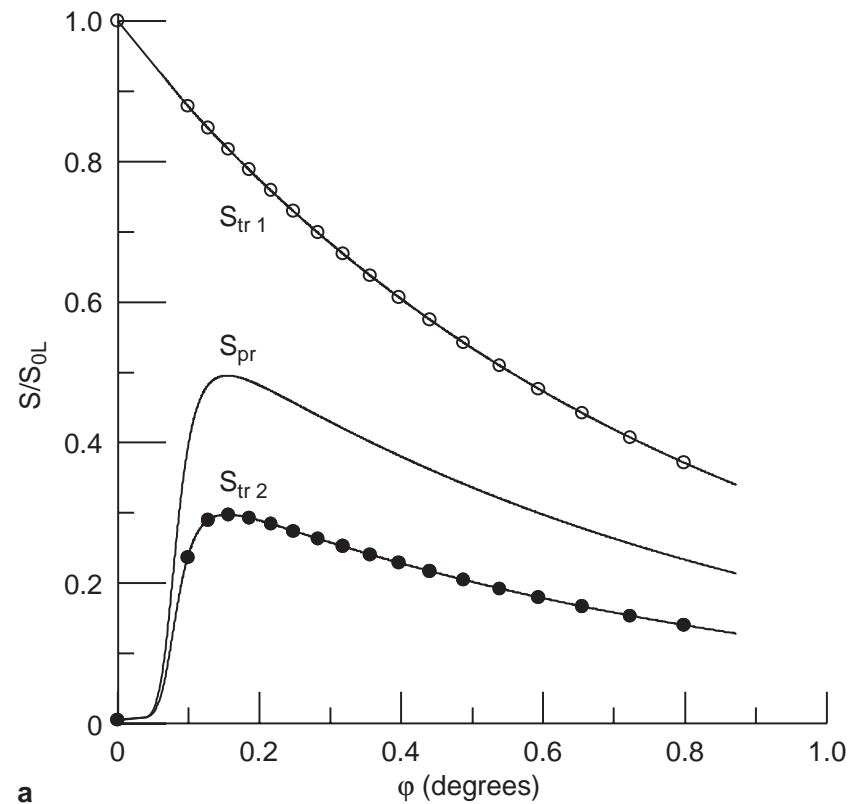

velocity because diffusion coefficient depends on $v$ as $D \propto v^{-2}$, thus at first electrons with small energy values reach the loss cone. The shift in the weak diffusion regime is bigger because in this case the loss cone empties immediately, while in the case of strong diffusion loss time depends on the electron velocity according to Eq. (3).

Examples of fluxes of trapped and precipitating electrons are presented in Figs. 3-4 as functions of $\varphi$ for different $\Delta v$ values in cases of strong (Fig. 3) and

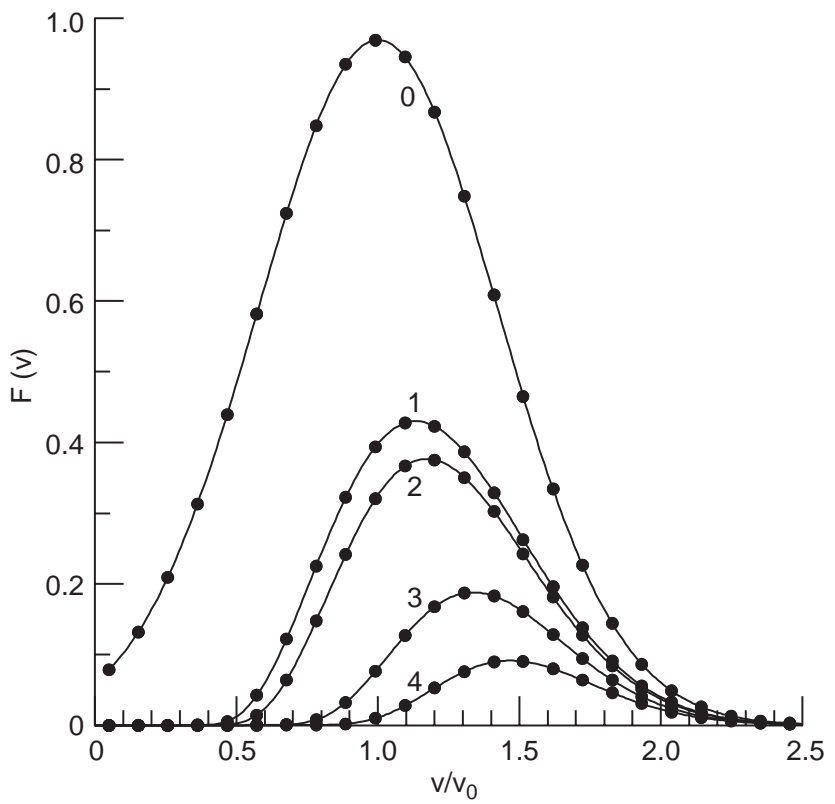

Fig. 2. The transformation of energetic electron distribution over energy $F(v)$ with increase in $\varphi$ in the weak diffusion case at equatorial plane. $\Delta v / v_{0}=0.6, \quad m v_{0}^{2} / 2=50 \mathrm{keV}, \mu_{c}^{-1}=160, D_{\max } \mu_{c}^{-1} \approx 0.5$, $v_{g \perp}=10^{2} \mathrm{~km} / \mathrm{s}, \quad \ln \left(D_{m} / D_{0}\right) \approx 10, \quad v_{0} / \gamma_{m}(0)=0.01, \quad \varphi_{0}=0$, $\varphi_{1} \approx 7.8^{\circ}, \varphi_{2} \approx 8.9^{\circ}, \varphi_{3} \approx 14.5^{\circ}, \varphi_{4} \approx 20.5^{\circ}$

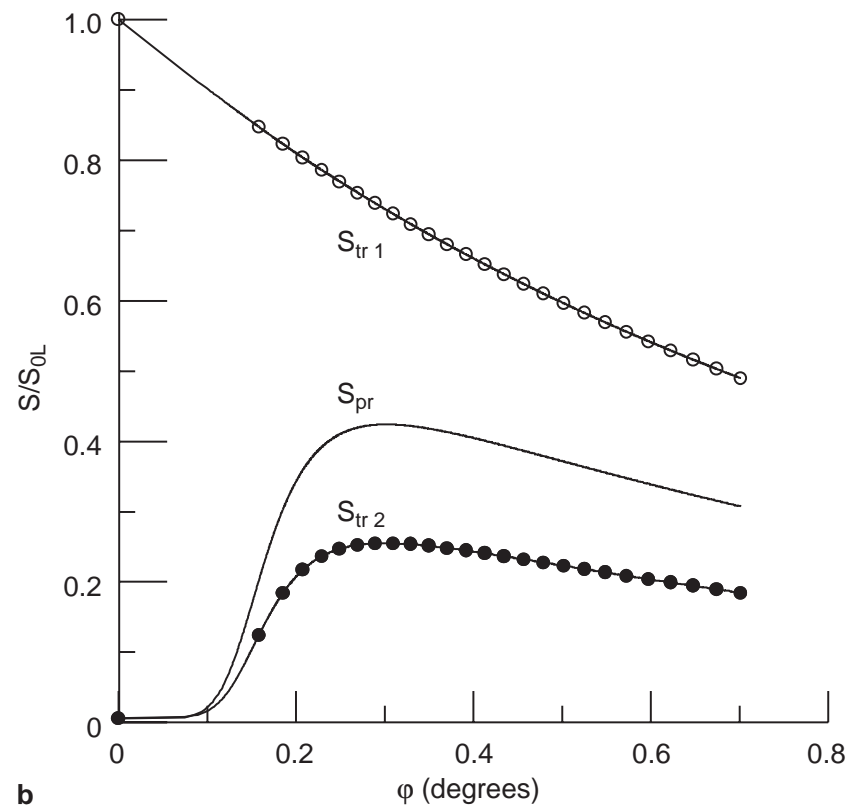

Fig. 3a, b. The dependence of fluxes of trapped and precipitated electron on $\varphi$ for strong diffusion for different initial spread over energies: a $\Delta v / v_{0}=0.3, \mathbf{b} \Delta v / v_{0}=0.6$, other parameters are as in Fig. 1 

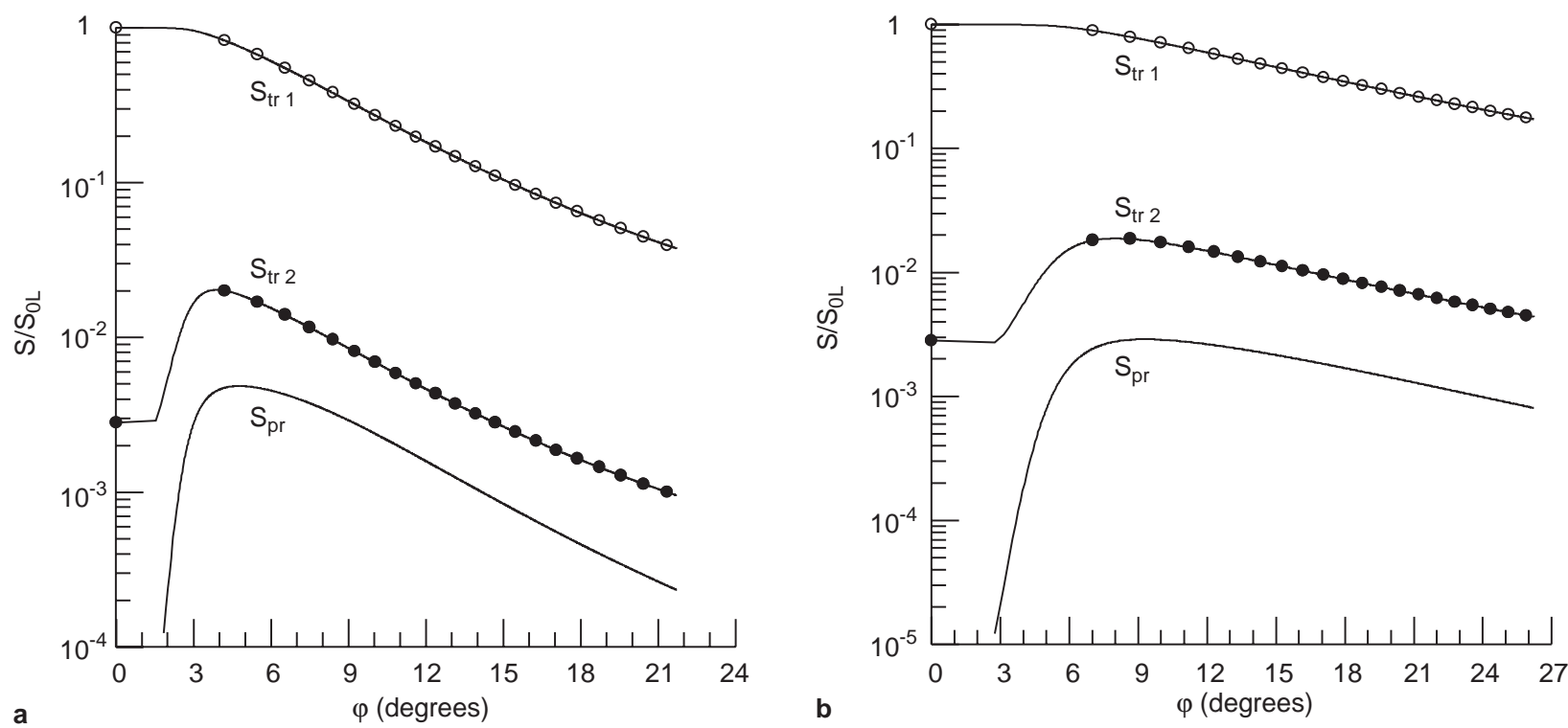

Fig. 4a, b. The dependence of fluxes of trapped and precipitated electrons on $\varphi$ for weak diffusion for different initial spread over energies: a $\Delta v / v_{0}=0.3, \mathbf{b} \Delta v / v_{0}=0.6$, other parameters are as in Fig. 2

weak (Fig. 4) diffusion. Qualitatively these dependencies are similar with changing of the initial velocity spread ( $\Delta v / v_{0}$ value) (compare also with results for $\delta$-function by Pasmanik et al., 1998). The dependencies of particle fluxes at low attitudes (curves $S_{t r 2}$ and $S_{p r}$ ) become smoother, with increase of $\Delta v / v_{0}$, and their maximum values decrease and maximum locations shift to a bigger $\varphi$ value. The energetic spectra of electron fluxes at low altitudes are given in Fig. 5 for strong diffusion and in Fig. 6 for weak diffusion.

The results of numerical analysis of the wave spectrum are presented in Figs. 7-8 for strong and weak diffusion regimes respectively. The sharp changes of the growth rate and wave spectral density as functions of frequency with strong diffusion are due to the process of fast isotropisation of the distribution function. It is clear that since the distribution of energetic particles are close to isotropic, the wave generation stops and the growth rate $\gamma$ has a negative value in almost all frequency ranges. Another situation is with weak diffusion when the energetic electron distribution is always sufficiently anisotropic and the growth rate $\gamma$ is always positive at low frequencies.

However, there are some difficulties in describing the transition between the cases of strong and weak diffusion. It is obvious that for weak diffusion the condition $K \ll 1$ is satisfied for all particles at any point on $\varphi$ axis. With strong diffusion the situation is different. Since $K \propto \Theta(\varphi) v^{-2}$ the strong diffusion condition $K \gg 1$ is not satisfied for particles with an energy greater than
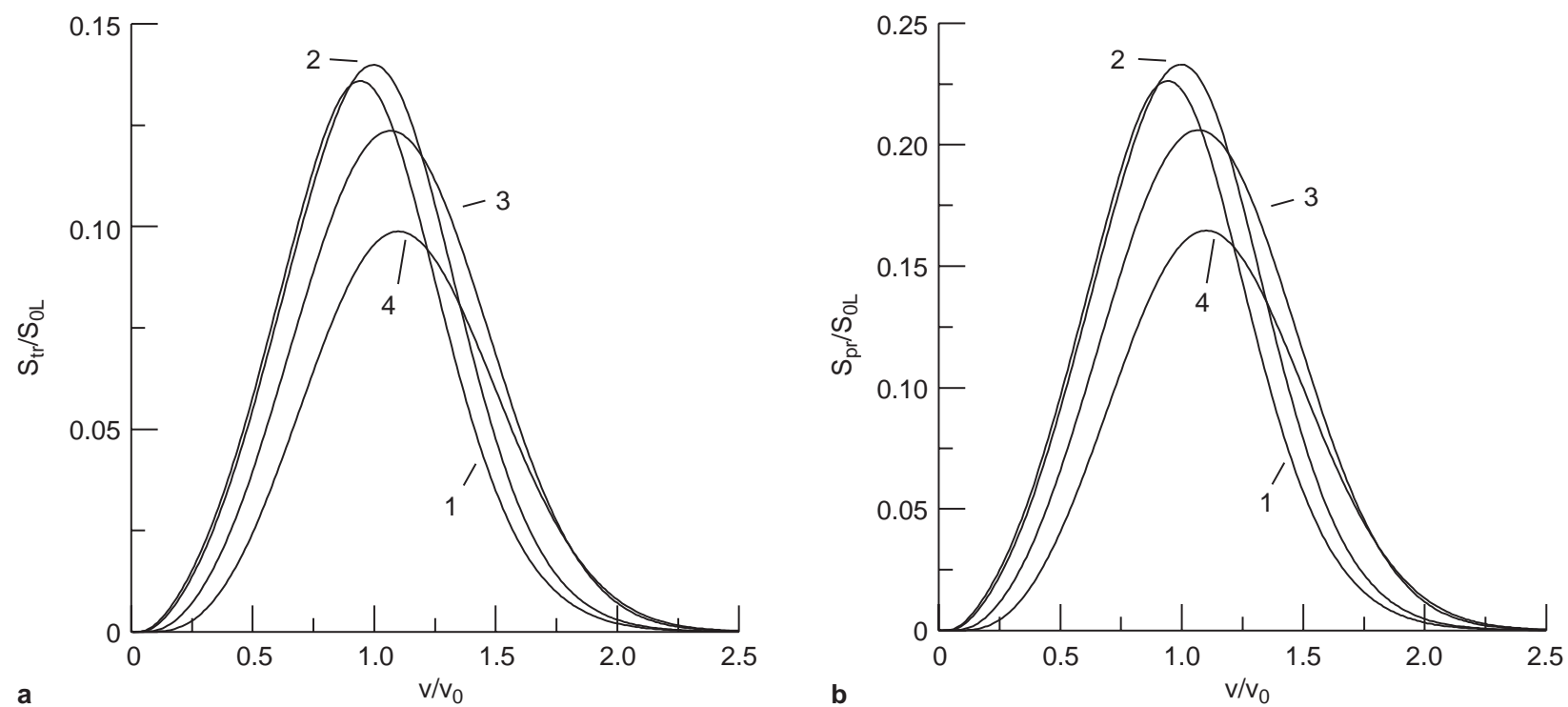

Fig. 5a, b. Energetic spectrum of a trapped and $\mathbf{b}$ precipitating energetic electron flux at low altitude for strong diffusion for different $\varphi$ values. $\Delta v / v_{0}=0.6, \varphi_{1} \approx 0.18^{\circ}, \varphi_{2} \approx 0.2^{\circ}, \varphi_{3} \approx 0.32^{\circ}, \varphi_{4} \approx 0.5^{\circ}$, other parameters are as in Fig. 1 

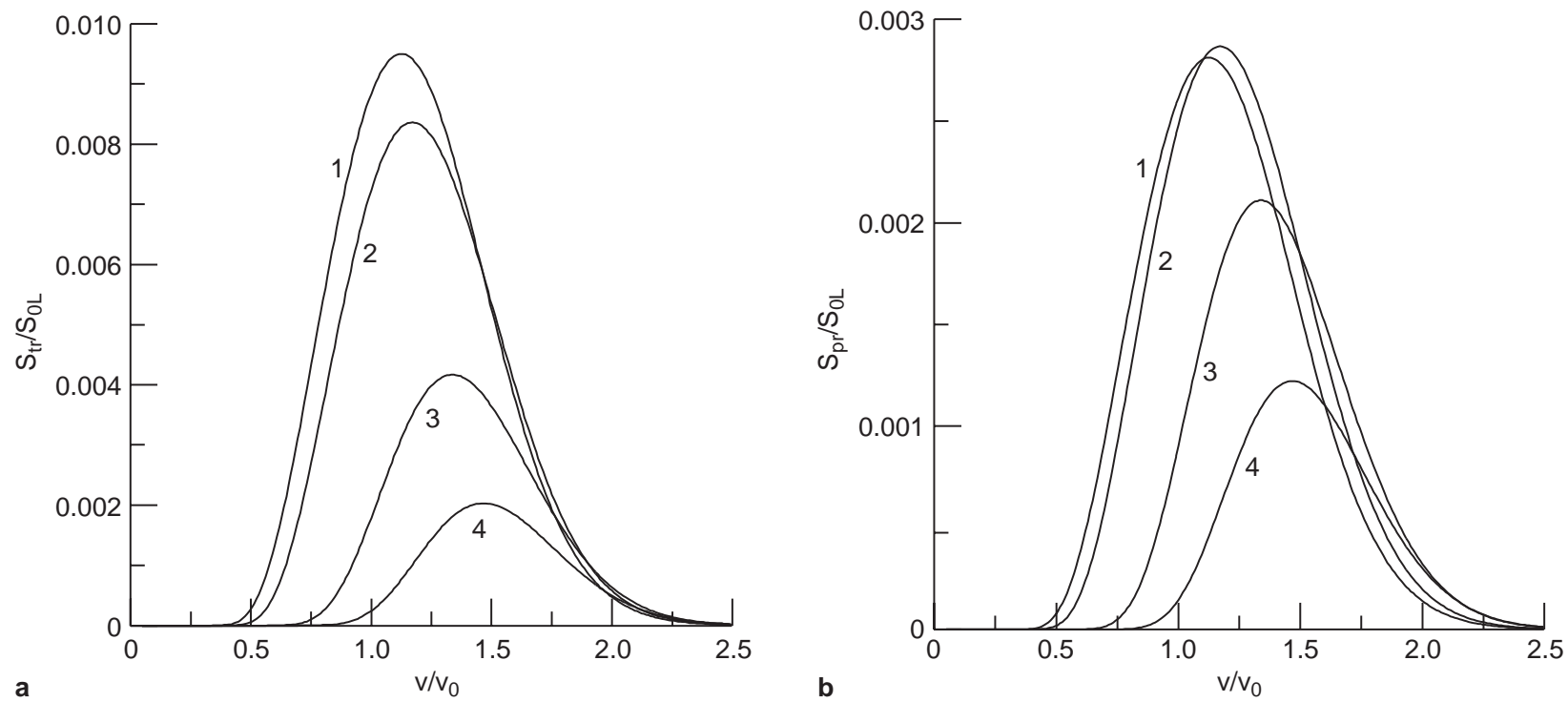

Fig. 6a, b. Energetic spectrum of $\mathbf{a}$ trapped and $\mathbf{b}$ precipitating energetic electron flux at low altitude for weak diffusion for different $\varphi$ values. $\Delta v / v_{0}=0.6, \varphi_{1} \approx 7.8^{\circ}, \varphi_{2} \approx 8.9^{\circ}, \varphi_{3} \approx 14.5^{\circ}, \varphi_{4} \approx 20.5^{\circ}$, other parameters are as in Fig. 2

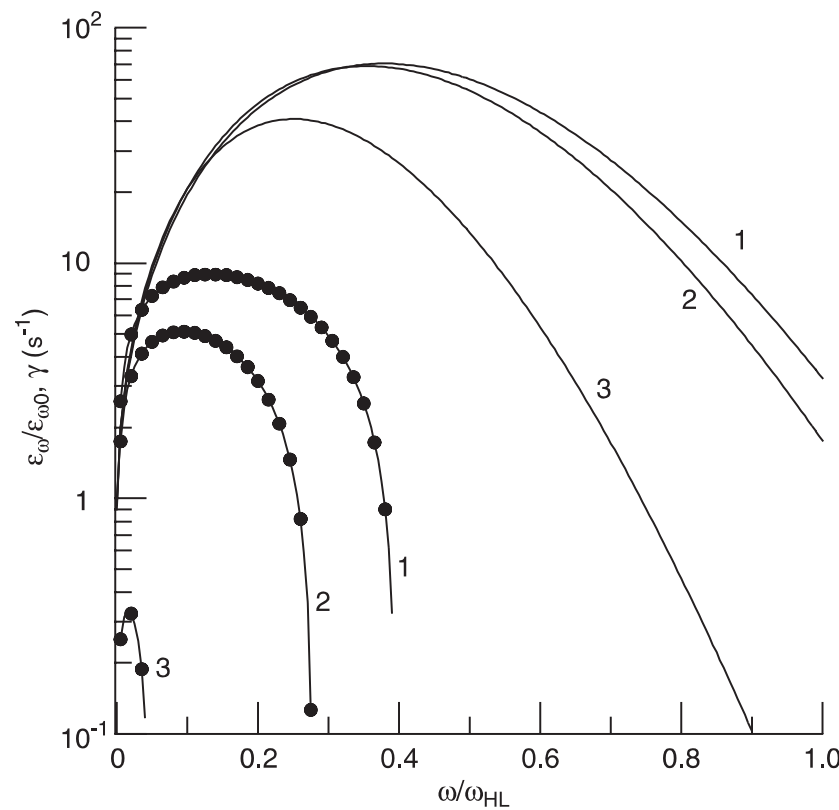

Fig. 7. The dependencies of growth rate (lines with dots) and intensity on frequency for strong diffusion for different $\varphi$ values $\Delta v / v_{0}=0.6$, $\varphi_{1} \approx 0.18^{\circ}, \varphi_{2} \approx 0.2^{\circ}, \varphi_{3} \approx 0.32^{\circ}$, other parameters are as in Fig. 1

some critical value. Moreover due to the decrease of $\Theta(\varphi)$ value as $\varphi$ increases the value of critical energy is also decreasing. Thus, a gradual transition from strong to weak diffusion regime occurs which may cause sufficient changes in the wave spectrum and precipitation process.

One way to take into account this transition is the following: for most particles the condition $K \gg 1$ is satisfied, and the solution for diffusion coefficient is obtained from the strong diffusion regime. Then it is possible to use this diffusion coefficient to find the solution for distribution function in cases of both strong

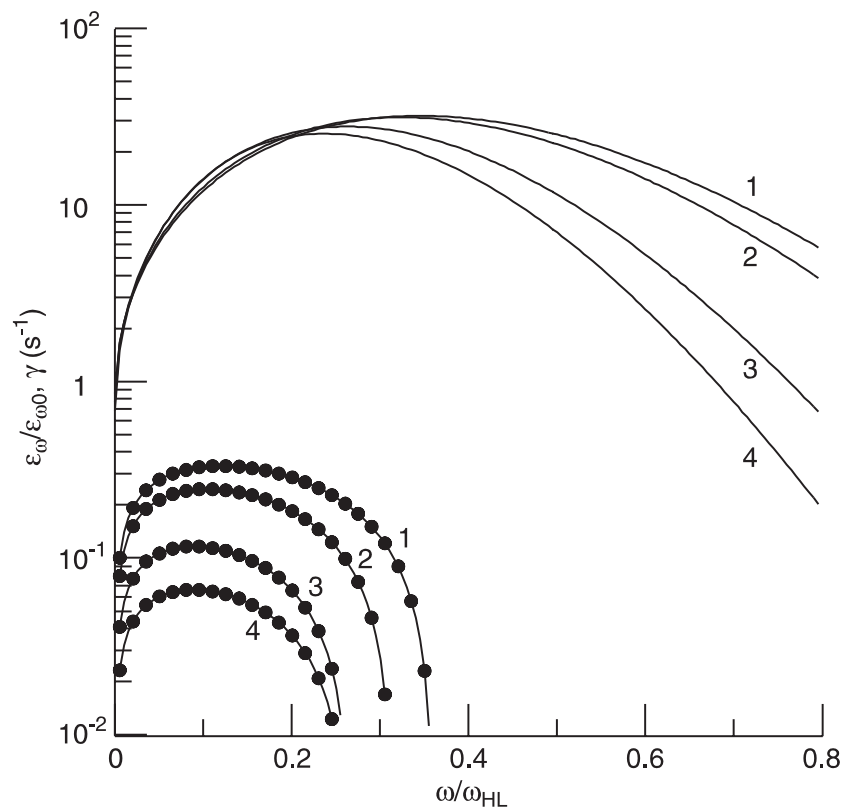

Fig. 8. The dependencies of growth rate (lines with dots) and wave intensity on frequency for weak diffusion for different $\varphi$ values $\Delta v / v_{0}=0.6, \varphi_{1} \approx 7.8^{\circ}, \varphi_{2} \approx 8.9^{\circ}, \varphi_{3} \approx 14.5^{\circ}, \varphi_{4} \approx 20.5^{\circ}$, other parameters as in Fig. 2

and weak diffusion and the boundary is determined from the relation $K(\varphi)=1$. But in an arbitrary case this method can be used only at the beginning of transition to a weak diffusion regime.

\section{Conclusions}

We have performed a further analysis of theoretical model of cyclotron wave-particle interactions allowing us to explain some quantitative characteristics of trapped and precipitating energetic particle fluxes ob- 
tained from experimental data (see for example Yahnina et al., 1996; Titova et al., 1997). Consideration of finite spread of energetic particles over energies and studying the wave spectrum provides a wider area of application of this model to the analysis of experimental data.

The investigation performed revealed new features which distinguish regimes of strong and weak diffusion. Namely, we found that with growth of the longitudinal coordinate the maximum of growth rate shifts rapidly to low frequencies for strong diffusion, and stays near the same location in weak diffusion regime. This causes a sufficiently different evolution of wave spectrum. On the other hand, energetic spectrum of particle fluxes at low altitudes moves to higher energies for weak diffusion, and remains actually at the same energies for strong diffusion.

As a further development of the model considered it would be useful to study more carefully the transition from strong to weak diffusion. Also it is necessary to improve the wave energy transfer equation by considereing the strict model for whistler wave propagation near the plasmapause, which serves as a wave-guide. This is an important problem, which includes the analysis of the eigenmodes' spatial structure as well as the refraction of group rays across the plasmapause wave-guide, and demands special consideration.
Acknowledgements. This work was supported by the Russian Foundation for Basic Research, grant 96-02-16473a.

\section{References}

Bespalov, P. A., and Trakhtengerts, V. Y., The cyclotron instability in the Earth radiation belts, in Ed. M. A. Leontovich, Reviews of Plasma Physics, vol 10, pp. 155-192, Plenum, New York, 1986.

Lyons, L. R., and Williams, D. J., Quantitative aspects of magnetospheric physics, D. Reidel, Dordrecht, Holland, 1984.

Pasmanik, D. L., Trakhtengerts, V. Y., Demekhov, A. G., Lyubchich, A. A., Titova, E. E., Yahnina, T., Rycroft, M. J., Manninen, J., and Turunen, T, A quantitative model for cyclotron wave-particle interactions at the plasmapause, Ann. Geophysicae, 16 (3), 322-330., (1998).

Titova, E. E., Yahnina, T. A., Yahnin, A. G., Gvozdevsky, B. B., Lyubchich, A. A., Trakhtengerts, V. Y., Demekhov, A. G., Horwitz, J. L., Lefeuvre, F., Lagoutte, D., Manninen, J., and Turunen, T., Strong localized variations of the low-altitude energetic electron fluxes in the evening sector near plasmapause, Ann. Geophysicae, 16(1) 25-33, 1997.

Yahnina, T. A., Titova, E. E., Yahnin, A. G., Gvozdevsky, B. B., Lyubchich, A. A., Trakhtengerts, V. Y., Demekhov, A. G., Horwitz, J. L., Manninen, J., and Turunen, T. Some features in the energetic electron precipitation pattern near the plasmapause in the evening sector, in Proc. XIX Seminar on Auroral Phenomena, pp. 70-72, Apatity, Russia. PGI, 1996. 